\title{
Impaired microvascular response to cholinergic stimuli in primary Sjögren's syndrome
}

\author{
László Kovács, Tamás Török, Ferenc Bari, Zsuzsanna Kéri, Attila Kovács, Éva Makula, \\ Gyula Pokorny
}

Department of Internal Medicine, Albert Szent-Györgyi Medical University, Szeged, Hungary

L Kovács

G Pokorny

Intensive Care Unit, Albert Szent-Györgyi Medical University

T Török

Z Kéri

Department of Physiology, Albert Szent-Györgyi Medical University

F Bari

Blood Transfusion Centre, Albert Szent-Györgyi Medical

University

A Kovács

Department of Radiology, Albert Szent-Györgyi Medical University

É Makula

Correspondence to: Dr L Kovács, 1st Department of Internal Medicine, Albert Szent-Györgyi Medical University, PO Box 469, Szeged, H-6701 Hungary

Acceptence for publication 13 August 1999

\begin{abstract}
Objective-Signs of a parasympathetic dysfunction have been revealed in primary Sjögren's syndrome (SS). Its role in the pathogenesis and the clinical picture of the disease is not clear. To investigate the responsiveness of SS patients to a cholinergic agonist, a model was used involving examination of the cutaneous microcirculation. The microvascular response to the administration of carbachol was measured, a muscarinic cholinergic agonist.
\end{abstract}

Methods-Twenty two SS patients and 12 controls were examined. Carbachol and $0.9 \%$ saline solution were administered intracutaneously into the forearm skin at two distinct places. Skin blood flow (SBF) in the injected areas was measured continuously before and for 10 minutes after the injections by means of a laser Doppler perfusion monitor. The increase in SBF in response to carbachol (dSBF), reflecting vasodilatation, was calculated by a formula including the baseline and the maximum SBF values after the injections of carbachol and saline solution.

Results-The vasodilatation was significantly lower in SS patients than in the controls (mean dSBF: 2.1 (range: 1.0-4.5) versus 3.3 (range: $1.7-7.6$ ), $p=0.02$ ). With non-responder patients defined as those in whom a smaller response was observed than in any of the controls, 11 of the 22 SS patients proved to be non-responders to carbachol. Comparisons of demographic, clinical and laboratory characteristics and HLA class II genotypes between responder and non-responder SS patients did not show any significant differences. Conclusions-A diminished or absent response to carbachol indicates a cholinergic dysfunction in SS patients. A disturbance in the neurotransmission at a receptorial or postreceptorial level is hypothesised. Unresponsiveness to cholinergic stimuli may contribute to exocrine insufficiency.

(Ann Rheum Dis 2000;59:48-53)

Dry mouth and dry eyes, the two fundamental symptoms of primary Sjögren's syndrome (SS), have previously been regarded solely as direct consequences of the loss of functioning glandular tissue caused by an autoimmune inflammatory process. However, this conception is now increasingly questioned. ${ }^{12}$ The symptoms of dryness and the quantitatively determined saliva and tear production may vary greatly during the course of the disease, even without treatment, and their spontaneous improvement is often observed for varying periods. A marked discrepancy has been reported between the degree of exocrine insufficiency and the histologically verified intensity of the inflammatory infiltration in the salivary glands. ${ }^{3}$ In human and animal experiments, several types of abnormalities of the autonomic nervous system have been demonstrated in $\mathrm{SS}^{4-10}$

The question of whether an impairment of parasympathetic innervation may play a part in the elicitation of exocrine insufficiency in SS remains an issue to be examined. Despite previous work, results of human functional studies on the parasympathetic nervous system in SS patients are by no means numerous. If a postulated cholinergic dysfunction does exist, it is not known whether it is caused by a central or a peripheral nervous system dysfunction, or whether it reflects a disorder of the parasympathetic innervation at a receptorial or postreceptorial level. Nor is it clear whether the acetylcholine transmitter system or a nonadrenergic, non-cholinergic transmitter (for example, vasoactive intestinal polypeptide (VIP)) system colocalised in the cholinergic nerve terminals is involved. ${ }^{11}$

For these reasons, we set out to examine the response to the administration of a muscarinic cholinergic agonist, carbachol, in SS patients and to compare it with that in healthy controls. To measure this response, we established a model involving examination of the cutaneous microcirculation. A cholinergic vasodilatory mechanism of the human skin blood vessels has long been verified. ${ }^{12-14}$ As this is similar in many respects to the cholinergic innervation of the salivary glands, ${ }^{13}$ we decided to use this experimental setting to test the hypothesis that a cholinergic dysfunction exists in SS patients.

\section{Methods}

PATIENTS AND CONTROLS

Twenty two primary SS patients (20 female and 2 male) were enrolled in the study. All were diagnosed as having SS by the European Community criteria ${ }^{15}$ at the Division of Autoimmune Diseases at the 1st Department of Internal Medicine, Albert Szent-Györgyi Medical University, Szeged, Hungary. The average age of the patients was (mean (SD)) 50.6 (13.2) years (33-60). The average time since the first symptom had appeared was (mean (SD)) 11.5 (5.2) years (4-24), while the average time since the establishment of the diagnosis was (mean 
(SD)) 9.1 (6.7) years (3-20). In 18 of the 22 patients, a minor salivary gland biopsy had been performed. In 17 of these 18 patients, histological examination revealed focal lymphocytic sialadenitis, meeting the histological criteria of SS, while in one patient a negative result was obtained. In this latter patient and in the four patients on whom no biopsy was performed, the appropriate number of other criteria for the diagnosis of SS was met. All patients were regularly followed up. To exclude other factors that might possibly influence the microcirculatory physiology, SS patients older than 60 years, those who had hypertension or clinical evidence of arteriosclerosis (coronary heart disease, arteriosclerotic cerebral disease or arteriosclerosis in other organs) or peripheral neuropathy, and those who regularly took $\beta$-adrenergic blockers, calcium channel blockers, pentoxyphylline, or other drugs with vasodilatory or anticholinergic properties, were regarded as not eligible for the study. For the same reason, the use of non-steroidal antiinflammatory drugs was suspended at least five days before the examinations. The patients were asked to refrain from smoking or the drinking of coffee on the day of the examination.

Twelve healthy, age and sex matched people were examined as controls. None of these subjects had any known illness, or were taking any regular medication. All gave their informed consent to the procedure and the study design was accepted by the Medical Ethics Board of Albert Szent-Györgyi Medical University.

EXAMINATION OF THE CUTANEOUS VASCULAR RESPONSE

During examinations, the patients and controls were in the supine position. The skin blood flow (SBF) was measured with a laser-Doppler flowmeter (Penflux, Perimed) attached to the skin on the flexor surface of the right forearm, and blood flow values were expressed in arbitrary units. ${ }^{16}$ Three ECG electrodes were attached to the chest and the heart rate was monitored continuously, as was the blood pressure by means of a photoplethysmographic blood pressure monitor (Finapres 2300, Ohmeda) attached to a finger of the right hand (beat to beat registration). The subjects were asked to lie at rest with their eyes closed. The room temperature was kept constant $\left(20^{\circ} \mathrm{C} \pm 1^{\circ} \mathrm{C}\right)$ and all disturbing factors were excluded. The baseline SBF was recorded for five minutes; the blood flow was stable by the end of this period and the SBF value measured at five minutes was defined as SBF basal. Then $0.1 \mathrm{ml}$ of carbachol (Miostat, Alcon, USA), a muscarinic receptor agonist, was injected intracutaneously into the forearm skin. As control, almost simultaneously, $0.1 \mathrm{ml}$ of $0.9 \%$ saline solution was injected similarly into the forearm skin at approximately $10 \mathrm{~cm}$ from the other injection site. SBF was measured simultaneously at the two injection sites for another 10 minutes and the highest deviations from the baseline flow values were designated SBF final.

All recorded data (heart rate, systolic and diastolic arterial pressure and SBF) were stored in a computer database and were analysed by means of a self developed software. The change in SBF in response to the injection of carbachol (dSBF) was calculated using the following formula:

$\mathrm{dSBF}=\frac{\text { SBFfinal, carbachol/SBFbasal, carbachol }}{\text { SBFfinal, saline/SBFbasal, saline }}$

Thus, dSBF is the ratio of the SBF values measured after and before the injection of carbachol divided by the same ratio for the control solution of physiological saline. This calculation allowed us to eliminate the absolute flow values and also to eliminate possible nonspecific microcirculatory effects of the intracutaneous injection. In every subject, the mean values of the RR intervals on the ECG, and the systolic, diastolic and mean arterial pressures were also recorded. The cutaneous vascular resistance (CVR) was calculated by dividing the mean arterial pressure by SBF, while dCVR was calculated with a formula analogous to that for dSBF.

\section{LABORATORY EXAMINATIONS}

Laboratory examinations were performed as follows: anti-SSA and anti-SSB antibodies: enzyme linked immunosorbent assay (Epignost, Leonding/Linz, Austria); antinuclear antibodies: indirect immunofluorescence assay, using rat liver as substrate; $\operatorname{IgM}$ rheumatoid factor: quantitative measurements by immunoturbidimetry (Boehringer Mannheim, Germany). HLA DRB1, DQA1 and DQB1 genotyping was performed by using methods described in detail by others. ${ }^{17-20}$ Schirmer's test and sialometry were performed within one year from the examinations in all SS patients. As sialometric examination, we assessed the unstimulated whole saliva production in 10 minutes. Skin dryness was scored on a scale $0-$ IV on the basis of the patients' reports of the severity of dry skin complaints and physical examination of the skin, as follows: grade 0 : no dry skin; grade I: a transient feeling of skin dryness not requiring treatment, and no objective signs of dry skin; grade II: a recurrent feeling of skin dryness requiring treatment with hydrating lotions, and objective signs of

Table 1 Occurrences of main organ manifestations and laboratory variables in primary Sjögren's syndrome patients $(n=22)$

\begin{tabular}{ll}
\hline Organ manifestations and laboratory variables & $\begin{array}{l}\text { Number of } \\
\text { patients (\%) }\end{array}$ \\
\hline Articular involvement ${ }^{\star}$ & $17(77)$ \\
Renal involvement† & $4(18)$ \\
Bronchitis sicca & $4(18)$ \\
Pulmonary fibrosis & $2(9)$ \\
Raynaud's phenomenon & $6(27)$ \\
Skin vasculitis & $3(14)$ \\
MALT lymphoma in parotid gland & $2(9)$ \\
Anaemiał & $6(27)$ \\
Hypergammaglobulinaemia\} $&{15(68)} \\
{\text { Antibody positivity }} &{ } \\
{\text { Anti-SSA }} &{14(64)} \\
{\text { Anti-SSB }} &{9(41)} \\
{\text { IgM rheumatoid factor }} &{18(82)} \\
{\text { Antinuclear antibody }} &{18(82)} \\
{ }$
\end{tabular}

${ }^{\star}$ Clinically evident arthritis or persistent arthralgia without objective physical signs. †Chronic tubulointerstitial nephritis or renal tubular acidosis. $¥$ Haemoglobin $<100 \mathrm{~g} / 1$ not attributable to causes other than SS. $\$ \gamma$ globulin $>16 \mathrm{~g} / 1$. 
Table 2 Comparison of certain haemodynamic variables in primary Sjögren's syndrome (SS) patients and controls recorded during the blood flow examinations

\begin{tabular}{|c|c|c|c|c|c|c|c|c|}
\hline Group & $\begin{array}{l}\text { SBFfinal,carbachol/ } \\
\text { SBFbasal,carbachol }\end{array}$ & $\begin{array}{l}\text { SBFfinal,salinel } \\
\text { SBFbasal,saline }\end{array}$ & $d S B F$ & $d C V R$ & Mean RR (msec) & $\begin{array}{l}\text { Mean SBP } \\
(m m \mathrm{Hg})\end{array}$ & $\begin{array}{l}\text { Mean } \mathrm{MBP} \\
(m m \mathrm{Hg})\end{array}$ & $\begin{array}{l}\text { Mean DBP } \\
\text { (mm } \mathrm{Hg})\end{array}$ \\
\hline SS patients & $1.89(1.05)^{\star}$ & $0.94(0.29)$ & $2.07(1.12) \dagger$ & $0.64(0.32) \ddagger$ & $827.9(109.6)$ & $132.4(22.0)$ & $95.9(16.5)$ & $76.3(14.9)$ \\
\hline Controls & $2.72(1.27)$ & $0.86(0.19)$ & $3.30(1.79)$ & $0.38(0.17)$ & $797.0(117.3)$ & $128.6(14.9)$ & $93.8(9.8)$ & $74.9(7.5)$ \\
\hline
\end{tabular}

Data shown as mean (SD). SBF: skin blood flow; CVR: cutaneous vascular resistance; RR: RR interval on ECG; SBP: systolic arterial blood pressure; MBP: mean arterial blood pressure; DBP: diastolic arterial blood pressure. SBF and CVR values are in arbitrary units. For the calculation of dSBF and dCVR, see the text (Methods section). ${ }^{\star} \mathrm{p}=0.048, \mathrm{tp}=0.019, \neq \mathrm{p}=0.013$.

decreased sweat production; grade III: a continuous feeling of skin dryness revealed by topical treatment, and severely dry skin with fine scaling; grade IV: a continuous feeling of skin dryness not revealed by topical treatment, and very severely dry skin with excoriations and mild loss of hair.

STATISTICAL ANALYSIS

Differences between means were calculated with an independent sample $t$ test, except for the comparison of the means of dSBF values, where Wilcoxon's $t$ test was applied because of the non-parametric distribution of the elements of the samples. For the comparisons of frequencies, Fisher's exact test was used, with Bonferroni's correction when the examined variable had more than two possible values (for example, HLA genotype). To investigate correlations with dSBF, Spearman's signed rank test or linear regression analysis was applied. Statistical calculations were performed with SPSS software. Values in the text are expressed as mean (SD). The level of significance was defined as $\mathrm{p}<0.05$.

\section{Results}

Table 1 gives details relating to the occurrences of the main systemic organ manifestations of SS and the various laboratory parameters in the 22 patients.

Table 2 reports the average values of the most important haemodynamic variables in the two groups recorded during the examinations of the cutaneous microcirculation. We present here the means of the changes in the local blood flow in response to carbachol

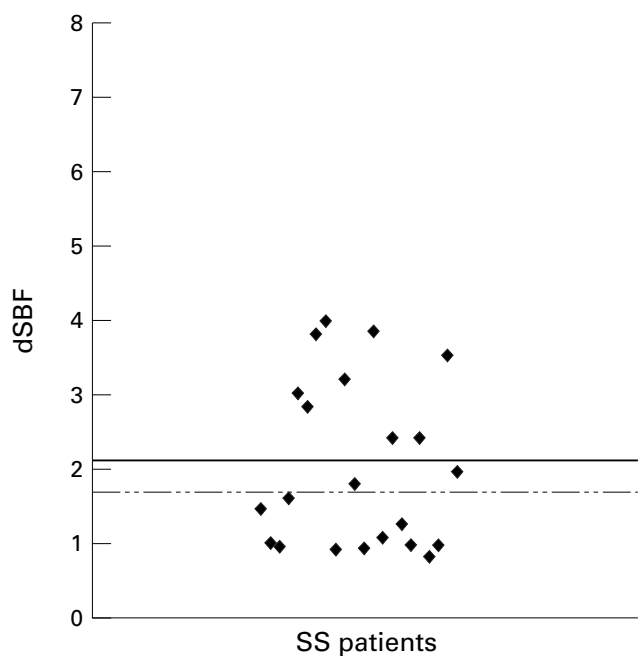

(SBFfinal,carbachol/SBFbasal,carbachol) and physiological saline solution (SBFfinal,saline/ SBFbasal,saline), the dSBF values (which are specific indicators of the cutaneous vasodilatation in response to the cholinergic agonist), and also the corresponding values of the change in CVR. The means of the continuously recorded values of the RR intervals on the ECG and of systemic arterial blood pressure are also included in table 2 .

In the event of a positive vascular reaction, the blood flow started to increase within 30 seconds after the injection of carbachol and reached a new steady state approximately 4-7 minutes later, then remaining unchanged until the end of the examination. In the controls, the average dSBF was 3.30 (1.79), which was significantly higher than the average dSBF in SS patients $(2.07(1.12))(p=0.019)$ (fig 1). In the controls, the dSBF values reflected a 1.66-7.6fold increase in microcirculatory blood flow after the injection of carbachol. However, in a relatively high proportion of the SS patients, the reaction to the administration of carbachol was small or virtually absent, while in other patients a marked vasodilatation was observed. We defined a positive microvascular reaction to carbachol in the SS patients as a dSBF value higher than the smallest dSBF value in the group of healthy controls. Following this definition, exactly half of the SS patients (11 of 22) could be considered to be nonresponders - that is, producing a less than 1.66-fold increase in the microcirculatory blood flow-while the other half of the patients were regarded as responders to carbachol on

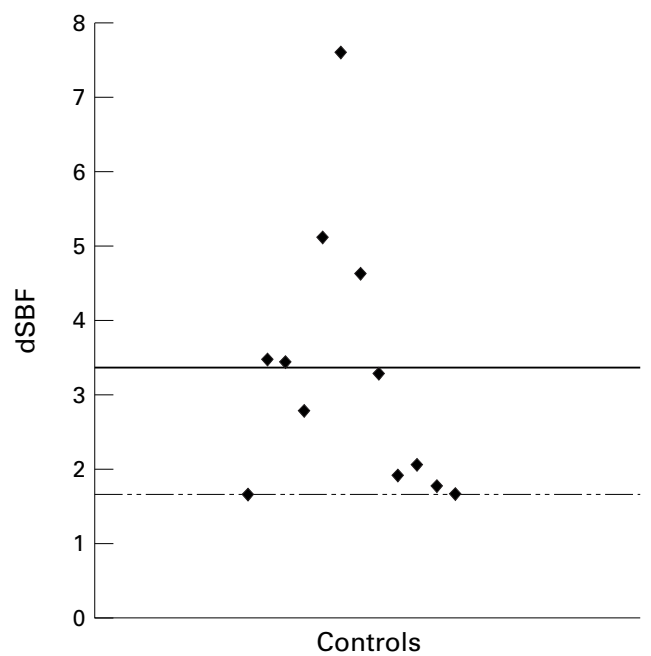

Figure 1 Distribution of dSBF values in primary Sjögren's syndrome (SS) patients and healthy controls. dSBF: change in skin blood flow in response to the injection of carbachol. Continuous lines indicate means. Values above the dashed lines indicate a positive response to carbachol. 
Table 3 Demographic characteristics and occurrences of certain organ manifestations of primary Sjögren's syndrome (SS) in carbachol responder and non-responder SS patients

\begin{tabular}{lll}
\hline & Responders $(n=11)$ & Non-responders $(n=11)$ \\
\hline Average age (y) & 51.0 & 50.3 \\
Average time since first symptom (y) & 11.8 & 11.2 \\
Articular involvement & 9 & 8 \\
Renal involvement & 3 & 1 \\
Bronchitis sicca & 3 & 1 \\
Pulmonary fibrosis & 1 & 1 \\
Raynaud's phenomenon & 5 & 1 \\
Skin vasculitis & 2 & 1 \\
MALT lymphoma in parotid gland & 2 & 0 \\
\hline
\end{tabular}

For organ manifestations, numbers indicate the number of involved patients. For the precise definitions of articular and renal involvement, see table 1 . No significant difference was found between the two subgroups. other, although, in view of the relatively small number of patients and the heterogeneity of the genotypes of the examined persons, a statistically significant difference is hardly to be expected (data not shown).

The drug treatment applied throughout the course of the illness was also surveyed. The proportions of patients treated with nonsteroidal anti-inflammatory drugs, hydroxychloroquine or corticosteroids were similar in the two subgroups. Two patients were receiving regular corticosteroid treatment and five hydroxychloroquine treatment at the time of the examination; the use of these drugs did not seem to influence the microcirculatory response to carbachol, as indicated by the similar proportions of users in the responder and nonresponder subgroups.

severity of shin drymess among the prinary Sjees of syndrome patients $(n=22)$

\begin{tabular}{llllll}
\hline Degree of skin dryness & 0 & I & II & III & IV \\
Number of patients & 5 & 4 & 6 & 7 & 0
\end{tabular}

For a precise description of the assessment of the severity of skin dryness, see the text (Methods section).

the basis of the pronounced vasodilatation (a more than 1.66-fold increase in SBF).

We examined whether there was any difference in certain demographic characteristics or in the frequencies of certain clinical organ involvements between the SS patients defined as non-responders or as responders to carbachol (table 3). The age of the patients and the time since the appearance of the first symptom of SS was similar in the two groups. Similarly, when tested by linear regression analysis, the above variables did not demonstrate any correlation with $\mathrm{dSBF}$ (data not shown). No statistically significant difference was revealed between the two groups in the occurrence of any of the main organ manifestations. However, it is noteworthy that all the examined organ involvements occurred more frequently in the responder patients (with the exception of pulmonary fibrosis, which occurred in one patient in each of the subgroups) (table 3). Dry skin is a common complaint in SS patients. In table 4 , we present the proportions of patients with varying degrees of severity of skin dryness. We checked whether there was a correlation between the reactivity of the cutaneous microcirculatory vessels to carbachol (dSBF) and the degree of skin dryness, which is predominantly a consequence of the insufficiency of the exocrine glands of the skin; the answer proved negative. The two subgroups were also comparable as concerns the average values of tear and saliva production (mean tear flow with Schirmer's test: 4.0 (2.12) $v 4.45$ (2.21) $\mathrm{mm} / 5$ minutes, unstimulated whole saliva flow: 0.34 $(0.28) v 0.32(0.28) \mathrm{ml} / 10$ minutes in the nonresponders and in the responders, respectively).

The frequencies of anti-SSA, anti-SSB, IgM RF and ANA positivity, and also the average levels of laboratory markers of a chronic autoimmune inflammatory process, in particular the erythrocyte sedimentation rate, fibrinogen concentration, anaemia and $\gamma$ globulin concentration, did not differ significantly in the two subgroups of primary SS patients. No HLA DR, DQA1 and DQB1 allele occurred more frequently in one subgroup than in the

\section{Discussion}

The purpose of this study was to determine the proportion of primary SS patients who demonstrate signs of a cholinergic dysfunction, and to evaluate whether there is a relation between the presence of a cholinergic dysfunction and certain clinical, serological and genetic characteristics of the disease. The importance of this question stems from the fact that the salivary glands are richly innervated by cholinergic nerve fibres, and, if the hypothesis that there is a disturbance in the cholinergic innervation proves true, this may provide new aspects as regards the pathogenesis of the sicca symptoms in SS. The issue of abnormalities in the parasympathetic nervous system in SS has arisen in previous human or animal studies in which various stages of the parasympathetic et $a l^{6}$ and Andonopoulos et al detected autonomic neuropathy, mainly affecting the parasympathetic nerves. Santavirta et $a l^{8}$ demonstrated that the salivary outputs of VIP (considered a marker of the parasympathetic innervation) and of neuropeptide Y (a marker of sympathetic innervation) were both increased as compared with healthy controls. They suggested that this phenomenon is associated with an altered response to stress in SS patients. In immunohistological examinations, Konttinen et $a l^{4}$ found that VIP containing parasympathetic fibres were absent from regions of the parotid glands where inflammatory cell infiltration and acinar atrophy were severe. Circulating antibodies against rat parotid M3 muscarinic cholinergic receptors were detected by Bacman et al. ${ }^{9}$ Törnvall et a $\bar{P}$ reported that tein kinase $\mathrm{C}$, a crucial member of the intracellular second messenger pathway of cholinergic neurotransmission, was deficient on the salias compared with controls. In contrast with healthy controls, the $\xi$ and $\beta_{\text {II }}$ isoforms were undetected on the myoepithelial cells in SS patients. These findings are suggestive of a postreceptorial disturbance of the cholinergic innervation.

In this study, we examined the cutaneous cholinergic vasodilatation in SS patients in response to the local administration of a innervation pathway were investigated. Mandl the expression of the $\beta_{\text {II }}$ and $\alpha$ isoforms of provary gland acinar epithelial cells in SS patients 
muscarinic cholinergic agonist, carbachol. Acetylcholine has been demonstrated to cause vasodilatation in healthy humans. ${ }^{13}$ Although the non-adrenergic-non-cholinergic transmitter systems probably play a more important part in the physiological vasoregulation in the skin than do the cholinergic nerves, receptors for acetylcholine can also be found in the cutaneous microvasculature. ${ }^{21}$ Carbachol has been demonstrated to induce phospholipase C dependent saliva secretion through stimulation of the muscarinic cholinergic receptors in human salivary acinar cells. ${ }^{22}{ }^{23}$ The same muscarinic receptors have been implicated in the cholinergic vasodilatation in human cutaneous arterioles in response to body heat stress. ${ }^{13}$ These findings provide a basis for our choice of an investigation of the cutaneous microcirculation as a model of the parasympathetic innervation of the salivary glands. Nevertheless, firm conclusions can be drawn only after examinations directly involving the salivary glands in SS patients.

We found that the average increase in cutaneous microcirculatory blood flow in response to carbachol was significantly smaller in the SS patients than in the healthy controls. In half of the SS patients, the reaction to a potent parasympathomimetic drug was virtually absent or markedly diminished. As vasodilatation is characterised physiologically by a decrease in vascular resistance, the CVR was also calculated by dividing the mean arterial pressure by SBF. As the arterial pressure remained virtually unchanged in all the examined persons during the examinations, an increase in SBF always reflected cutaneous vasodilatation in this experimental setting.

The above finding lends support to previous results suggesting a disturbance in the parasympathetic autonomic nervous system in SS. As our experiments involved the administration of a muscarinic receptor agonist directly to the examined target organ, the detected unresponsiveness favours the hypothesis that a dysfunction may exist at a receptorial or post-receptorial level. To test whether a receptorial dysfunction may result from a structural abnormality of the receptor protein caused by an abnormal genetic coding, we determined the HLA DRB1, DQA1 and DQB1 genotype profiles in our study patients. Certain HLA alleles (most importantly HLA-DR3) are known to occur more frequently in SS patients than in the general population. However, the hypothesis that an acetylcholine receptor pathology may be determined by a genetic predisposition remains an open question, as our relatively small patient population does not allow firm conclusions in this regard. Antireceptor antibodies, similarly to myasthenia gravis, may be other possible causes of a receptor dysfunction. Indeed, antibodies against rat $\mathrm{M}_{3}$ muscarinic receptors have been detected in SS patients, ${ }^{9}$ though the existence and possible role of anti-receptorial antibodies against human muscarinic receptors have not been verified.

It has been hypothesised that an interaction may occur in the salivary glands between the infiltrating inflammatory cells and certain neuropeptides. ${ }^{4}$ In contrast, another hypothesis suggests that the loss of trophic stimuli from the parasympathetic nerves in the salivary glands might be a cause of acinar cell atrophy, and this may contribute to the decreased saliva production. ${ }^{4}$ Our results relating to investigation of a target organ distant from the areas of lymphocytic inflammation suggest that a disorder of the cholinergic receptors may not be restricted to the exocrine glands, but may be a more widely occurring phenomenon, and that this defect is possibly independent of the local inflammatory process. Further investigations may yield results of general importance as concerns the interactions between the nervous system and the immune system.

As a considerable proportion of the SS patients displayed a fundamentally insignificant reaction to carbachol, we could divide our population of SS patients into responders and non-responders to the cholinergic agent. The occurrence of unresponsiveness did not correlate with the age of the patients, the disease duration, the presence of a specific organ manifestation (including vascular manifestations of the disease) or of an autoantibody, nor the severity of the functional impairment of the salivary and lachrymal glands. However, all the organ involvements proved to be more common in the responder patients than in the nonresponders. Confirmation of this latter finding with statistically significant results is first necessary before an explanation can be suggested.

In summary, we found that half of the examined SS patients proved to be non-responders to the administration of the cholinergic receptor agonist carbachol. Whether this unresponsiveness also relates to the clinically involved exocrine glands, and the precise mechanism of the disorder in the cholinergic innervation, possibly at a receptorial or postreceptorial level, are features that remain to be clarified.

1 Price EJ, Venables PJW. The etiopathogenesis of Sjögren's syndrome. Semin Arthritis Rheum 1995;25:117-33.

2 Fox RI, Maruyama T. Pathogenesis and treatment of Sjögren's syndrome. Curr Opin Rheumatol 1997;9:393-9.

3 Andoh Y, Shimura S, Sawai T, Sasaki H, Takishima T, Shirato K. Morphometric analysis of airways in Sjögren's syndrome. Am Rev Respir Dis 1993;148:1358-62.

4 Konttinen YT, Hukkanen M, Kemppinen P, Segerberg M, Sorsa T, Malmström M, et al. Peptide-containing nerves in labial salivary glands in Sjögren's syndrome. Arthritis Rheum 1992;35:815-20.

5 Törnwall J, Konttinen YT, Tuominen RK, Törnwall M. Protein kinase C expression in salivary gland acinar epithelial cells in Sjögren's syndrome. Lancet 1997;349: 1814-15.

6 Mandl T, Jacobsson L, Lilja B, Sundkvist G, Manthorpe R. Disturbances of autonomic nervous function in primary Sjögren's syndrome. Scand J Rheumatol 1997;26:401-6.

7 Andonopoulos AP, Christodoulou J, Ballas C, Bounas A, Alexopoulos D. Autonomic cardiovascular neuropathy in Sjögren's syndrome. A controlled study. J Rheumatol 1998;25:2385-8.

8 Santavirta N, Konttinen YT, Törnwall J, Segerberg M, Santavirta S, Matucci-Cerinic M, et al. Neuropeptides of the autonomic nervous system in Sjögren's syndrome. Ann Rheum Dis 1997;56:737-40.

9 Bacman S, Sterin Borda L, Camusso JJ, Arana R, Hubscher $\mathrm{O}$, Borda E. Circulating antibodies against rat parotid gland $\mathrm{M}_{3}$ muscarinic receptors in primary Sjögren's syndrome. Clin Exp Immunol 1996;104:454-9.

10 Konttinen YT, Sorsa T, Hukkanen M, Segerberg M, Kuhlefelt-Sundström M, Malmström M, et al. Topology of innervation of labial salivary glands by protein gene product 9.5 (PGP 9.5)- and synaptophysin immunoreactive nerves in patients with Sjögren's syndrome. J Rheumative nerves in patient
tol 1992;19:30-7. 
11 Lundberg JM, Anggard A, Fahrenkrug J, Hökfelt T, Mutt V. Vasoactive intestinal polypeptide in cholinergic neurons of exocrine glands: functional significance of coexisting transmitters for vasodilatation

12 Coffman JD, Cohen RA. Cholinergic vasodilator mechanism in human fingers. Am J Physiol 1987;252:H594-7.

13 Kellogg DL, Jr, Pérgola PE, Piest KL, Kosiba WA, Crandal $\mathrm{CG}$, Grosmann $\mathrm{M}$, et al. Cutaneous active vasodilation in humans is mediated by cholinergic nerve cotransmission. Circ Res 1995;77:1222-8.

14 Taylor WF, Johnson JM, Kosiba WA, Kwan CM. Cutaneous vascular responses to isometric handgrip exercise. J Appl Physiol 1989;66:1586-92

15 Vitali C, Moutsopoulos HM, Bombardieri S and the European Community Study Group on Diagnostic Criteria for Sjögren's Syndrome. The European Community Study Sroup on Diagnostc Criteria for Siögren's syndry Study Group on Diagnostic Criteria for Sjogren's syndrome. Seninvolvement in Sjögren's syndrome. Ann Rheum Dis 1994; 53:637-47.

16 Török T, Bari F, Paprika D, Rudas L, Kardos A, Gingl Z. Short-term monitoring of the vascular resistance of the human skin microvasculature. Acta Physiol Hung 1998;85. $153-62$.
17 Davies KE. Human genetic diseases. A practical approach. Oxford: IRL Press, 1986:56-7.

18 Ota $M$. HLA-DRB1 genotyping by modified PCR-RFLP method combined with group-specific primers. Tissue Antigens 1992;39:187-202.

19 Ota M, Seki T, Namura N, Sugimura K, Mizuki N, Fukushima $\mathrm{H}$, et al. Modified PCR-RFLP method for HLA-DPB1 and DQA1 genotyping. Tissue Antigens 1991;38:60-71.

20 Mercier B, Ferec C, Dufosse F, Huart JJ. Improvement in HLA-DQB typing by PCR-RFLP: Introduction of a constant restriction site in one of the primers for digestion control. Tissue Antigens 1992;40:86-9.

21 Zar, M.A. Acetylcholine, atropine and related cholinergic and anticholinergics. In: Greaves, MW, Shuster S, eds: Pharmacology of the skin I. Berlin: Springer, 1989:331-45.

22 Komabayashi T, Yakata A, Izawa T, Fujinami H, Suda K, Tsuboi M. Mechanism of carbachol-stimulated diacylglycerol formation in rat parotid acinar cells. Eur J Pharmacol 1992;225:209-16.

23 Soltoff SP, Toker A. Carbachol, Substance P and phorbol ester promote the tyrosine phosphorylation of protein kinase $\mathrm{C} \delta$ in salivary gland epithelial cells. J Biol Chem 1995;270:13490-5. 